\title{
The effect of thin lenticule versus thick lenticule (a cut-off value of 55 micrometer) on the outcome of SMILE in low myopia
}

\author{
Zaid Yousif Hameed Shukur
}

College of Medicine, University of Kufa, Najaf, Iraq.

Corresponding author: Zaid Yousif Hameed Shukur (E-mail: zaid.shukur@uokufa.edu.iq)

(Submitted: 19 July 2020 - Revised version received: 29 July 2020 - Accepted: 17 August 2020 - Published online: 30 October 2020)

\begin{abstract}
Objectives: In the current study, the aim was to evaluate the effect difference in lenticule thickness small incision lenticule extraction (SMILE) cases $(\leq 55 \mu \mathrm{m}$ versus $>55 \mu \mathrm{m}$ ) on the visual outcome in a cohort of 61 patients with low myopia ( $\leq 3.0$ diopters)

Methods: The current prospective cohort study included 61 patients undergoing SMILE refractive procedure during the period extending from November, 142019 to June 14, 2020, a total of 99 eyes. The study was conducted in Ibsar ophthalmology surgery centre «private medical centre» in the wholly Najaf city, mid-Euphrates region of Iraq. The cases enrolled in the study were all operated by the same ophthalmologist. According to lenticule thickness, eyes were classified into two groups, ( $\leq 55 \mu \mathrm{m}$ versus $>55 \mu \mathrm{m})$. The main outcome variable was visual acuity postoperative within two short period of time, 1 week and 3 months.

Results: Comparison of visual acuity between patients with lenticule thickness of $\leq 55 \mu \mathrm{m}$ and patients with lenticule thickness of $>55 \mu \mathrm{m}$ are shown in Table 6. There was no significant difference in UCVA $(P=1.000)$, significant difference in BCVA (better with lenticule thickness of $\leq 55 \mu \mathrm{m})(P=0.037)$, no significant difference in VAAS $(P=0.065)$, and no significant difference in VA3M $(P=0.599)$.

Conclusion: Thin lenticule of less than or equal 55 micrometer to as low as 41 micrometer can be chosen for SMILE with efficacy and safety comparable to that of thicker lenticule provided that the operation is done by well trained and skilled refractive surgeon.

Key words: Thin lenticule, SMILE, low myopia
\end{abstract}

\section{Introduction}

One of the common causes of refractive errors globally is myopia, and myopic astigmatism. ${ }^{1-3}$ This refractive error can be permanently corrected by the well-known laser refractive surgeries such as laser-assisted in situ keratomileusis (LASIK) or small incision lenticule extraction (SMILE) ${ }^{4-7}$. In 2008, SMILE was introduced for the first time to be a novel refractive surgical approach. ${ }^{8}$ A paradigm shift has been marked by refractive lenticule extraction in the refractive surgery field from the well-known and practiced flap-based corneal ablative techniques to flapless extraction method of femtosecond laser-produced intrastromal lenticules. ${ }^{9}$

The extraction of an intrastromal lenticule has been described by Sekundo et al following lifting a flap in 2008, and the procedure has been developed to the SMILE, a procedure in which a small $2-5-\mathrm{mm}$ side cut incision is made through which the refractive lenticule is extracted. ${ }^{8,10,11}$

As of the time this paper is being prepared, femtosecond LASIK and SMILE are considered to be the most widely accepted procedures by both refractive surgeons and myopic patients for the correction of refractive errors. ${ }^{12}$ The procedure of SMILE involves the production of an intrastromal lenticule using femtosecond laser, and then this lenticule is going to be extracted through a small peripheral corneal incision, thereby, considering it as a minimally invasive refractive corneal surgery., 13 Although, pervious reports have shown comparable efficacy, with respect to long-term visual acuity results, between femto-LASIK and SMILE, the later operation is superior for having a number of advantages including greater corneal sensitivity, superior biomechanics, less induction of higher-order aberrations and fewer dry eye symptoms. ${ }^{14-17}$ Probably, the major disadvantage of SMILE is the delayed optimum recovery of visual acuity following surgery in comparison with LASIK. ${ }^{11}$, ${ }^{18-20}$ SMILE offers precise visual quality and acuity and because of the enhanced patient satisfaction, the technique is increasingly being preferred for the correction of refractive errors associated with myopia and myopic astigmatism. ${ }^{9}$

Nevertheless, SMILE has a steep learning curve and this is a challenge for beginners. ${ }^{21,}{ }^{22}$ In order to familiarize the beginner surgeon to delicate procedures of SMLIE, a stepwise approach is needed and this involves observation, wetlab training, followed by flap-based refractive lenticule extraction (pseudo-SMILE). ${ }^{8,21,23}$ During early learning phase, a high rate of intraoperative complications may be seen due to difficult lenticule dissection and extraction..$^{21,24,25}$ In order to ease the process of lenticule extraction easier and to minimize complications, various modifications of the surgical procedure have been developed as SMILE is gaining global acceptance among occular surgeons. ${ }^{9}$

The scanning trajectory of the femtosecond laser is being modified in order to improve Visual outcome following SMILE. A number of approaches have been tried in order to improve short-term post-operative visual acuity results following SMILE including lowering laser energy levels, changing cap thickness, and intraoperative cap repositioning. ${ }^{26-29}$

On the other hand, the effect of the difference between diameters of the cap and lenticule on early visual and refractive outcome has been investigated by some authors. ${ }^{30}$

In the current study, the aim was to evaluate the effect difference in lenticule thickness ( $\leq 55 \mu \mathrm{m}$ versus $>55 \mu \mathrm{m}$ ) on the visual outcome in a cohort of 61 patients with low myopia.

\section{Patients and Methods}

The current prospective cohort study included 61 patients undergoing SMILE during the period extending from November 14, 2019 to June 14, 2020, a total of 99 eyes. The 
study was conducted in Ibsar ophthalmology surgery centre "private medical centre» in the wholly Najaf city, mid-Euphrates region of Iraq. The cases enrolled the study were all operated on by the same ophthalmologist.

The main inclusion criterion was patients with low myopia ( $\leq-3.0$ diopter). Other inclusion criteria were stable refraction in the last 2 years, and normal corneal topography. Exclusion criteria were eyes with severe dryness, cataract, uveitis, or progressive corneal degeneration. According to lenticule thickness, eyes were classified into two groups, $(\leq 55 \mu \mathrm{m}$ versus $>55$ $\mu \mathrm{m})$. The main outcome variable was visual acuity post-operative within two short period of time, 1 week and 3 months.

The study was approved by the institutional ethical approval committee associated with faculty of medicine and health directorate, in addition, a verbal consent was obtained from every participant.

Regular pattern of corneal topography was confirmed, before the SMILE procedure, by OCULUS Pentacam ${ }^{\circledast}$ HR from OCULUS Inc, USA. In all enrolled patients, the mesopic (4 lux) pupil diameter was $\leq 6.5 \mathrm{~mm}$ and the residual thickness of the stromal bed was $>250 \mu \mathrm{m}$. Performance of SMILE was done using the VisuMax femtosecond laser (Carl Zeiss Meditec, Jena, Germany) with a $500 \mathrm{kHz}$. In order to produce the four cleavage planes (lenticule horizontal cut, lenticule vertical cut, the cap cut, and the peripheral external opening cut), the femtosecond laser with an energy of $130 \mathrm{~nJ}$ was utilized.

All participants were subjected to a complete ophthalmic examination pre-operatively and at day 1 , week 1 , and month 3 , post-operatively. Visual acuity was assessed using a Snellen chart at $6 \mathrm{~m}$ in a well-illuminated room. Examination also included slit-lamp biomicroscopy, dilated fundoscopy examination, and corneal topography.

Data were analyzed using SPSS for Windows (Statistical Package for Social Sciences, ver. 23.0, SPSS Inc., Chicago, Illinois, USA) software. Quantitative data were expressed as mean, range, and standard deviation, while, categorical data were expressed as number and percentage. Independent samples $t$-test was used to study difference in means between the two groups, while, Yates correction and Fischer exact tests were used to evaluate association between categorical variables. The level of significance was considered at $P \leq 0.05$, and high significance at $P \leq 0.01$.

\section{Results}

The current study included 61 patients with low myopia $(\leq 3$ diopter) with a mean age of $27.67 \pm 7.75$ years and an age range of 18-56 years. The study enrolled $23(37.7 \%)$ males and 38 $(62.3 \%)$ females. Total number of eyes included in this study was 99, 52 (52.5\%) right and 47 (47.5\%) left, as shown in Table 1.

According to lenticule thickness, eyes were categorized into two groups, $(\leq 55 \mu \mathrm{m}$ versus $>55 \mu \mathrm{m}$. Baseline uncorrected visual acuity of patients enrolled in the current study categorized according to lenticule thickness into ( $\leq 55$ versus $>55 \mu \mathrm{m}$ ) was shown in Table 2 . The mean uncorrected visual acuity of eyes with thinner lenticule ( $\leq 55 \mu \mathrm{m}$ thickness) was $6 /(34.95 \pm 22.70)$ and that of eyes with thicker lenticule ( $>55$ $\mu \mathrm{m}$ thickness $)$ was $6 /(15.81 \pm 5.50)$; the difference was highly significant, as shown in Table 2 . These visual acuity results assessed retrospectively.

Best corrected visual acuity of patients enrolled in the current study categorized according to lenticule thickness into
( $\leq 55$ versus $>55 \mu \mathrm{m}$ ) was shown in Table 3 . The mean visual acuity of eyes with $\leq 55 \mu \mathrm{m}$ thickness was $6 /(6.76 \pm 1.17)$ and that of eyes with $>55 \mu \mathrm{m}$ thickness was $6 /(8.59 \pm 4.38)$; the difference was not significant $(P=0.075)$, as shown in Table 3 .

Visual acuity 1 week after surgery of patients enrolled in the current study categorized according to lenticule thickness into $(\leq 55$ versus $>55 \mu \mathrm{m})$ are shown in Table 4 . The mean uncorrected visual acuity of eyes with $\leq 55 \mu \mathrm{m}$ thickness was $6 /(6.74 \pm 0.99)$ and that of eyes with $>55 \mu \mathrm{m}$ thickness was $6 /$ $(8.16 \pm 3.24)$; the difference was not significant $(P=0.063)$, as shown in Table 4

Uncorrected visual acuity 3 months after surgery of patients enrolled in the current study categorized according to lenticule thickness into ( $\leq 55$ versus $>55 \mu \mathrm{m}$ ) are shown in Table 5 . The mean uncorrected visual acuity of eyes with $\leq 55$ $\mu \mathrm{m}$ thickness was $6 /(7.08 \pm 1.66)$ and that of eyes with $>55 \mu \mathrm{m}$ thickness was $6 /(7.34 \pm 2.15)$; the difference was not significant $(P=0.625)$, as shown in Table 5 .

Comparison of visual acuity between patients with lenticule thickness of $\leq 55 \mu \mathrm{m}$ and patients with lenticule thickness of $>55 \mu \mathrm{m}$ are shown in Table 6 . There was no significant difference in UCVA (uncorrected visual acuity) $(P=1.000)$, significant difference in BCVA (better with lenticule thickness of $\leq 55 \mu \mathrm{m})(P=0.037)$, no significant difference in VAAS (visual acuity after surgery) $(P=0.065)$ and no significant difference in VA3M (visual acuity 3 months after surgery) $(P=0.599)$. In order to produce the four cleavage planes (lenticule horizontal cut, lenticule vertical cut, the cap cut, and the peripheral external opening cut), the femtosecond laser with an energy of 130 nJ was utilized (Fig 1).

The beginning of the femtosecond laser from outside toward inside to create the lenticule lower surface which is called the lenticule interphase (green arrow). The peripheral cut ring represents the surrounding vertical cut of the lenticule periphery to be then separated from the rest of the stroma. The upper surface femtosecond laser cut of the lenticlue beginning from the center towards the far periphery which is called the cap interphase (blue arrow), The last femtosecond laser cut which is the surface incision cut at the periphery of the cap interphase (red arrow)

Table 1. Characteristics of patients enrolled in this study.

\begin{tabular}{ll}
\hline Characteristic & Value \\
\hline $\begin{array}{l}\text { Number of cases } \\
\text { Age (years) }\end{array}$ & 61 \\
$\quad$ Mean \pm SD & \\
$\quad$ Range (minimum-maximum) & 38 (18-56) \\
Gender & \\
$\quad$ Male, $n(\%)$ & $23(37.7 \%)$ \\
$\quad$ Female, $n(\%)$ & $38(62.3 \%)$ \\
Eye $\quad$ & \\
Total & 99 \\
OD, $n(\%)$ & $52(52.5 \%)$ \\
OS, $n(\%)$ & $47(47.5 \%)$ \\
\hline
\end{tabular}

SD: standard deviation; n: number of cases; 0D: right eye; OS: left eye. 


\begin{tabular}{|c|c|c|c|}
\hline \multirow[b]{2}{*}{ UCVA } & \multirow{2}{*}{$\begin{array}{c}\text { Total } \\
n=99\end{array}$} & \multicolumn{2}{|c|}{ Thickness group } \\
\hline & & $\begin{array}{c}\leq 55 \\
n=19\end{array}$ & $\begin{array}{c}>55 \\
n=80\end{array}$ \\
\hline $6 / 9.5$ & $12(12.1 \%)$ & 2 (10.5 \%) & 10 (12.5\%) \\
\hline $6 / 12$ & 27 (27.3\%) & $3(15.8 \%)$ & 24 (30.0\%) \\
\hline $6 / 15$ & $17(17.2 \%)$ & $1(5.3 \%)$ & 16 (20.0\%) \\
\hline $6 / 18$ & 20 (20.2\%) & $3(15.8 \%)$ & 17 (21.3\%) \\
\hline $6 / 24$ & 12 (12.1\%) & $1(5.3 \%)$ & 11 (13.8\%) \\
\hline $6 / 36$ & $3(3.0 \%)$ & $1(5.3 \%)$ & $2(2.5 \%)$ \\
\hline $6 / 60$ & $8(8.1 \%)$ & 8 (42.1%) & $0(0.0 \%)$ \\
\hline $\begin{array}{l}\text { Mean } \\
\pm S D\end{array}$ & $6 /(19.48 \pm 13.28)$ & $6 /(34.95 \pm 22.70)$ & $6 /(15.81 \pm 5.50)$ \\
\hline Range & $6 / 9.5-6 / 60$ & $6 / 9.5-6 / 60$ & $6 / 9.5-6 / 36$ \\
\hline$P$ & & $\begin{array}{c}<0.001 \dagger \\
\mathrm{HS}\end{array}$ & \\
\hline
\end{tabular}

$\boldsymbol{n}$ : number of cases; SD: standard deviation; UCVA: uncorrected visual acuity; $†$ independent samples t-test; $\mathrm{HS}$ : highly significant at $\mathrm{P} \leq 0.01$
Table 4. Visual acuity one week after surgery of patients enrolled in the current study categorized according to lenticule thickness into ( $\leq 55$ versus $>55 \mu \mathrm{m}$ ).

\begin{tabular}{cccc}
\hline & \multicolumn{3}{c}{ Thickness group } \\
\hline VAAS & Total & $\mathbf{5} 5 \mathbf{5}$ & $>\mathbf{5 5}$ \\
& $\boldsymbol{n = 9 9}$ & $\boldsymbol{n = 1 9}$ & $\boldsymbol{n = 8 0}$ \\
\hline $6 / 5$ & $1(1.0 \%)$ & $0(0.0 \%)$ & $1(1.3 \%)$ \\
$6 / 6$ & $44(44.4 \%)$ & $11(57.9 \%)$ & $33(41.3 \%)$ \\
$6 / 7.5$ & $32(32.3 \%)$ & $7(36.8 \%)$ & $25(31.3 \%)$ \\
$6 / 9$ & $1(1.0 \%)$ & $0(0.0 \%)$ & $1(1.3 \%)$ \\
$6 / 9.5$ & $7(7.1 \%)$ & $1(5.3 \%)$ & $6(7.5 \%)$ \\
$6 / 12$ & $9(9.1 \%)$ & $0(0.0 \%)$ & $9(11.3 \%)$ \\
$6 / 15$ & $3(3.0 \%)$ & $0(0.0 \%)$ & $3(3.8 \%)$ \\
$6 / 19$ & $1(1.0 \%)$ & $0(0.0 \%)$ & $1(1.3 \%)$ \\
$6 / 24$ & $1(1.0 \%)$ & $0(0.0 \%)$ & $1(1.3 \%)$ \\
Mean \pm SD & $6 /(7.88 \pm 2.99)$ & $6 /(6.74 \pm 0.99)$ & $6 /(8.16 \pm 3.24)$ \\
Range & $(5-24)$ & $(6-9.5)$ & $(5-24)$ \\
$P$ & & $0.063 \dagger$ & \\
& & $N S$ &
\end{tabular}

n: number of cases; SD: standard deviation; VAAS: visual acuity one week after surgery; + : independent samples t-test; $N$ S: not significant at $P>0.05$.

\begin{tabular}{|c|c|c|c|}
\hline \multirow[b]{2}{*}{ BCVA } & \multirow{2}{*}{$\begin{array}{c}\text { Total } \\
n=99\end{array}$} & \multicolumn{2}{|c|}{ Thickness group } \\
\hline & & $\begin{array}{c}\leq 55 \\
n=19\end{array}$ & $\begin{array}{c}>55 \\
n=80\end{array}$ \\
\hline $6 / 6$ & $52(52.5 \%)$ & $12(63.2 \%)$ & $40(50.0 \%)$ \\
\hline $6 / 7.5$ & $21(21.2 \%)$ & $5(26.3 \%)$ & $16(20.0 \%)$ \\
\hline $6 / 9.5$ & $9(9.1 \%)$ & 2 (10.5 \%) & 7 (8.8 \%) \\
\hline $6 / 12$ & 10 (10.1\%) & $0(0.0 \%)$ & $10(12.5 \%)$ \\
\hline $6 / 15$ & $2(2.0 \%)$ & $0(0.0 \%)$ & $2(2.5 \%)$ \\
\hline $6 / 19$ & $3(3.0 \%)$ & $0(0.0 \%)$ & $3(3.8 \%)$ \\
\hline $6 / 24$ & $1(1.0 \%)$ & $0(0.0 \%)$ & $1(1.3 \%)$ \\
\hline $6 / 30$ & $1(1.0 \%)$ & $0(0.0 \%)$ & $1(1.3 \%)$ \\
\hline Mean $\pm S D$ & $6 /(8.24 \pm 4.03)$ & $6 /(6.76 \pm 1.17)$ & $6 /(8.59 \pm 4.38)$ \\
\hline Range & $6 /(6-30)$ & $6 /(6-9.5)$ & $6 /(6-30)$ \\
\hline$P$ & & $\begin{array}{c}0.075 \dagger \\
\text { NS }\end{array}$ & \\
\hline
\end{tabular}

n: number of cases; SD: standard deviation; BCVA: best visual acuity; t independent samples t-test; $\mathrm{NS}$ : not significant at $\mathrm{P}>0.05$.
Table 5. Visual acuity 3 months after surgery of patients enrolled in the current study categorized according to lenticule thickness into ( $\leq 55$ versus $>55 \mu \mathrm{m}$ ).

\begin{tabular}{cccc}
\hline & & \multicolumn{2}{c}{ Thickness group } \\
\cline { 3 - 4 } VA3M & Total & $\mathbf{5}$ & $\mathbf{5 5 5}$ \\
& $\boldsymbol{n = 9 9}$ & $\boldsymbol{n = 1 9}$ & $\boldsymbol{n = 8 0}$ \\
\hline $6 / 5$ & $4(4.0 \%)$ & $0(0.0 \%)$ & $4(5.0 \%)$ \\
$6 / 6$ & $51(51.5 \%)$ & $11(57.9 \%)$ & $40(50.0 \%)$ \\
$6 / 7.5$ & $24(24.2 \%)$ & $5(26.3 \%)$ & $19(23.8 \%)$ \\
$6 / 9$ & $1(1.0 \%)$ & $0(0.0 \%)$ & $1(1.3 \%)$ \\
$6 / 9.5$ & $11(11.1 \%)$ & $2(10.5 \%)$ & $9(11.3 \%)$ \\
$6 / 12$ & $6(6.1 \%)$ & $1(5.3 \%)$ & $5(6.3 \%)$ \\
$6 / 15$ & $2(2.0 \%)$ & $0(0.0 \%)$ & $2(2.5 \%)$ \\
Mean & $6 /(7.29 \pm 2.06)$ & $6 /(7.08 \pm 1.66)$ & $6 /(7.34$ \\
\pm SD & & $6 /(6-12)$ & $6 /(5-15)$ \\
Range & $6 /(5-15)$ & $0.625 \dagger$ & \\
$P$ & & NS & \\
\hline
\end{tabular}

n: number of cases; SD: standard deviation; VA3M: visual acuity 3 months after surgery; + : independent samples t-test; NS: not significant at $P>0.05$ 


\begin{tabular}{|c|c|c|c|c|}
\hline \multirow{2}{*}{\multicolumn{2}{|c|}{ Visual acuity }} & \multicolumn{2}{|c|}{ Thickness group } & \multirow[b]{2}{*}{$\mathbf{P}$} \\
\hline & & $\leq 55$ & $>55$ & \\
\hline \multirow{2}{*}{ UCVA } & $\geq 6 / 9.5$ & 2 (10.5 \%) & $10(12.5 \%)$ & \multirow{2}{*}{$\begin{array}{c}1.000 \mathrm{Y} \\
\mathrm{NS}\end{array}$} \\
\hline & $<6 / 9.5$ & $17(89.5 \%)$ & 70 (87.5 \%) & \\
\hline \multirow{2}{*}{ BCVA } & $\geq 6 / 9.5$ & 19 (100.0\%) & $63(78.8 \%)$ & \multirow{2}{*}{$\begin{array}{c}0.037 \mathrm{~F} \\
\mathrm{~S}\end{array}$} \\
\hline & $<6 / 9.5$ & 0 (0.0\%) & 17 (21.3 \%) & \\
\hline \multirow{2}{*}{ VAAS } & $\geq 6 / 9.5$ & 19 (100.0\%) & $66(82.5 \%)$ & \multirow{2}{*}{$\begin{array}{c}0.065 \mathrm{~F} \\
\text { NS }\end{array}$} \\
\hline & $<6 / 9.5$ & $0(0.0 \%)$ & $14(17.5 \%)$ & \\
\hline \multirow{2}{*}{ VA3M } & $\geq 6 / 9.5$ & $18(94.7 \%)$ & 73 (91.3 \%) & \multirow{2}{*}{$\begin{array}{c}0.599 Y \\
\text { NS }\end{array}$} \\
\hline & $<6 / 9.5$ & $1(5.3 \%)$ & 7 (8.8 \%) & \\
\hline
\end{tabular}

n: number of cases; UCVA: uncorrected visual acuity; BCVA: best corrected visual acuity; VAAS: visual acuity after surgery; VA3M: visual acuity 3 months after surgery; Y: Yates correction; F: Fischer exact test; NS: not significant at P> 0.05 ; S: significant at $P \leq 0.05$

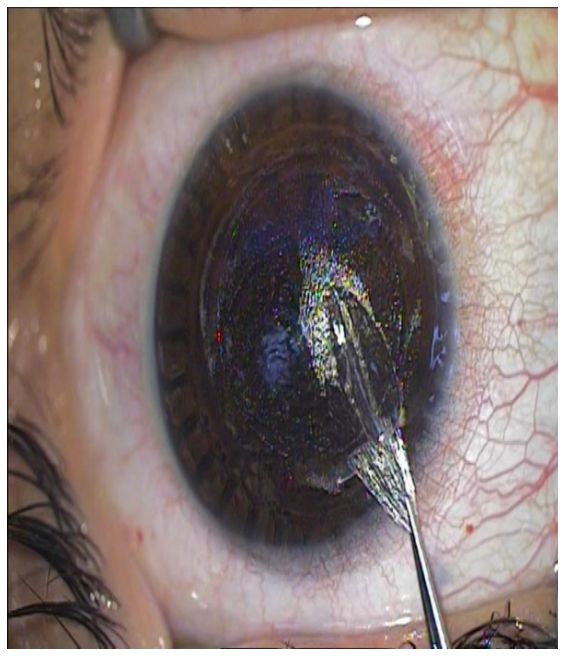

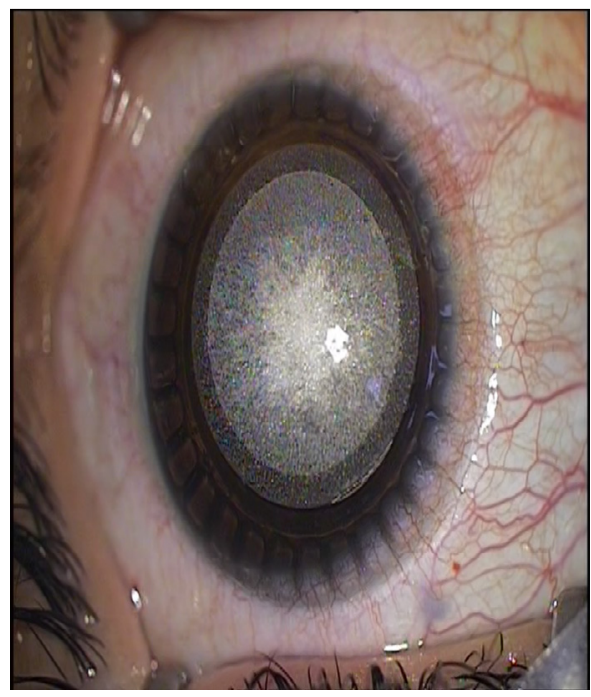

Fig. 1. This figure shows the different stages of the femtosecond laser applied during docking procedure for the correction of myopia or myopic astigmatism.

\section{Fig. 2. Through the peripheral opening the surgeon is accessible to introduce the dissector to separate the lenticule on both upper and lower surfaces and then removing it out of the pocket.}

\section{Discussion}

Laser refractive surgery field has been revolutionized following the introduction of femtosecond laser. This laser type has been widely used in LASIK and it resulted in the development of newer refractive surgery technique which uses single platform rather than the known two platforms for LASIK and this technique was named femtosecond lenticule extraction (FLEx). ${ }^{7,31}$ The later technique has also the advantage of avoiding corneal stromal ablation which is a necessary step in FLEx; however, it was similar to LASIK in that it needs formation of a corneal flap. ${ }^{31}$ The newly introduced laser refractive technique, SMILE, has the advantages of formation of corneal stromal pocket rather than corneal flap of old techniques, since it utilizes a small peripheral corneal incision in order to extract the lenticule. ${ }^{32}$ Because of minimal disruption of the peripheral collagen networks in the anterior stroma, the corneal stability will be preserved following SMILE much better than following oldest operations, LASIK and FLEx, in addition to avoidance of possible injury to the subbasal nerve plexus. ${ }^{33,}{ }^{34}$ Nowadays, SMILE is regarded as the standard of care for surgical correction of refraction errors by most ocular surgeons. ${ }^{8}$

Acar B. T. and Acar, in $2017^{30}$, compared the results of SMILE procedure between two groups of patients depending on the cap lenticule diameter difference (CLDD) and found that "In SMILE, $0.4 \mathrm{~mm}$ CLDD is associated with better visual outcome than $1.0 \mathrm{~mm}$ and that Narrow CLDD should be considered in SMILE to increase the visual acuity particularly in the early post-operative period". 
A study published in 2018, carried out by Siedlecki et al. ${ }^{35}$ was aiming at reporting the effect of increasing minimum lenticule thickness on the efficacy and safety and SMILE in patients with low myopia (up to -3.50 diopters). In the later study, thick lenticule group was compared in terms of visual and refractory outcome to standard thickness group and the results obtained pointed out to that "Increasing minimum lenticule border thickness seems to improve the safety and efficacy of SMILE in low myopia”. In the current study, we were able to show that there seems to be no significant difference in the efficacy of SMILE with thin lenticule, in the contrary to the belief of Siedlecki et al.$^{35}$, since it was noticed in the present study that the visual acuity of patients with thin lenticule was similar, and sometimes even better, than that of patients with thicker lenticule.

It seems that experienced hand can do SMILE with results that are of sufficient efficacy and safety when lenticule is as thin as $<55 \mu \mathrm{m}$ and this will increase the scope of selected myopic patients in need to do refractive surgery with thinner corneas as the minimum lenticule thickness we chose to preserve much residual stromal thickness to the acceptable limits.

\section{Conclusion}

Thin lenticule of less than or equal $55 \mu \mathrm{m}$ to as low as $41 \mu \mathrm{m}$ can be chosen for SMILE with efficacy and safety removal in one piece comparable to that of thicker lenticule provided that the operation is done by well-trained and skilled ocular surgeon hand to avoid tear of the lenticule and extract it as one piece (Fig. 2), and we can increase the scope of myopic patients in need to do SMILE procedure by using thinner SMILE lenticule and preserve the stromal tissue to the acceptable residual stromal thickness.

\section{Acknowledgment}

The author have no conflicts of interest

The author have no supported funding,

The author have no financial disclosure

The author of the current study would like to express deep thanks and appreciation to all patients participating in the study.

\section{References}

1. N Warner, "Update on myopia," Curr Opin Ophthalmol. 2016;27:402-406.

2. Wu PC, Huang HM, Yu HJ, Fang PC, Chen CT. Epidemiology of myopia. Asia Pac J Ophthalmol (Phila). 2016;5(6):386-393.

3. Foster PJ, Jiang Y. Epidemiology of myopia. Eye (Lond). 2014;28(2):202-208

4. GL Ehlke and RR Krueger, "Laser vision correction in treating myopia," AsiaPac J Ophthalmol. 2016;5:434-437

5. A Agca, A Demirok, Y Yıldırım et al., "Refractive lenticule extraction (ReLEX) through a small incision (SMILE) for correction of myopia and myopic astigmatism: Current perspectives," Clin Ophthalmol. 2016;10:1905-1912.

6. Kuryan J, Cheema A, Chuck RS. Laser-assisted subepithelial keratectomy (LASEK) versus laser-assisted in-situ keratomileusis (LASIK) for correcting myopia. Cochrane Database Syst Rev. 2017;2(2):CD011080.

7. Ganesh S, Brar S, Arra RR. Refractive lenticule extraction small incision lenticule extraction: A new refractive surgery paradigm. Ind J Ophthalmol. 2018;66(1):10-19.

8. Sekundo W, Kunert K, Russmann C, et al. First efficacy and safety study of femtosecond lenticule extraction for the correction of myopia: six-month results. J Cataract Refract Surg. 2008;34(9):1513-1520.
9. Titiyal JS, Kaur M, Shaikh F, Gagrani M, Brar AS, Rathi A. Small incision lenticule extraction (SMILE) techniques: Patient selection and perspectives. Clin Ophthalmol. 2018;12:1685-1699.

10. Sekundo W, Kunert KS, Blum M. Small incision corneal refractive surgery using the small incision lenticule extraction (SMILE) procedure for the correction of myopia and myopic astigmatism: Results of a 6 month prospective study. Br J Ophthalmol. 2011;95(3):335-339.

11. Shah R, Shah S, Sengupta S. Results of small incision lenticule extraction: All-in-one femtosecond laser refractive surgery. J Cataract Refract Surg. 2011;37(1):127-137.

12. M Liu, Y Chen, D Wang et al., "Clinical outcomes after SMILE and femtosecond laser-assisted LASIK for myopia and myopic astigmatism: A prospective randomized comparative study," Cornea. 2016:35:210-216.

13. E Chansue, M Tanehsakdi, S Swasdibutra, and C McAlinden, "Efficacy, predictability and safety of small incision lenticule extraction (SMILE),"Eye Vision. 2015:2:14

14. DZ Reinstein, TJ Archer, and M Gobbe, "Small incision lenticule extraction (SMILE) history, fundamentals of a new refractive surgery technique and clinical outcomes," Eye Vision. 2014:1:3.

15. Y Zhang, Q Shen, Y Jia, D Zhou, and J Zhou, "Clinical outcomes of SMILE and FS-LASIK used to treat myopia: A meta-analysis," J Refract Surg. 2016:32:256-265.

16. MJ Ye, CY Liu, RF Liao, G. Zy, BY Zhao, and Y Liao, "SMILE and wavefrontguided LASIK out-compete other refractive surgeries in ameliorating the induction of high-order aberrations in anterior corneal surface,"J Ophthalmol. 2016;2016:Article ID 8702162, 7 pages.

17. WT Cai, QY Liu, CD Ren et al., "Dry eye and corneal sensitivity after small incision lenticule extraction and femtosecond laser-assisted in situ keratomileusis: a meta-analysis," Int J Ophthalmol. 2017;10:632-638.

18. R Shah and S Shah, "Effect of scanning patterns on the results of femtosecond laser lenticule extraction refractive surgery," J Cataract Refractive Surg. 2011;37:1636-1647

19. A Vestergaard, A Ivarsen, S Asp, and JQ Hqjortdal, "Femtosecond (FS) laser vision correction procedure for moderate to high myopia: A prospective study of ReLex ${ }^{\circledast}$ flex and comparison with a retrospective study of FS-laser in situ keratomileusis," Acta Ophthalmol. 2013;91:335-362.

20. K Kamiya, K Shimizu, A lgarashi, and H Kobashi, "Effect of femtosecond laser setting on visual performance after small incision lenticule extraction for myopia," Br J Ophthalmol. 2015;99:1381-1387.

21. Titiyal JS, Kaur M, Rathi A, et al. Learning curve of small incision lenticule extraction: Challenges and complications. Cornea. 2017;36(11):1377-1382

22. Pradhan K, Reinstein D, Carp G, et al. Learning curve for small incision lenticule extraction (SMILE) of a novice corneal laser refractive surgeon evaluated by procedure time for consecutive cases. European Society of Cataract \& Refractive Surgeons. 2013. ESCRS website. Available from: http://escrs.org/amsterdam2013/programme/free-papers-details. asp?id=15825\&day $=0$.

23. Ang M, Mehta JS, Chan C, et al. Refractive lenticule extraction: Transition and comparison of 3 surgical techniques. J Cataract Refract Surg. 2014;40(9):1415-1424.

24. Ivarsen A, Asp S, Hjortdal J. Safety and complications of more than 1500 small-incision lenticule extraction procedures. Ophthalmology. 2014;121(4):822-828.

25. Ramirez-Miranda A, Ramirez-Luquin T, Navas A, Graue-Hernandez EO. Refractive lenticule extraction complications. Cornea. 2015;34(Suppl 10):S65-S67.

26. R Shetty, R Shroff, L Kaweri, C Jayadev, MK Kummelil, and A Sinha Roy, "Intraoperative cap repositioning in small incision lenticule extraction (SMILE) for enhanced visual recovery," Curr Eye Res. 2016;41:1532-1538.

27. M He, W Wang, H Ding, and X Zhong, "Comparison of two cap thickness in small incision lenticule extraction: $100 \mu$ m versus $160 \mu$ m," PLoS One. 2016;11, article e0163259.

28. YW. Ji, M Kim, DS Y. Kang et al., "Lower laser energy levels lead to better visual recovery after small-incision lenticule extraction: Prospective, randomized clinical trial," Am J Ophthalmol. 2017;179:159-170.

29. D Donate and R Thaëron, "Lower energy levels improve visual recovery in small incision lenticule extraction (SMILE),"J Refractive Surg. 2016;32:636642

30. Acar BT and Acar S. Effect of cap-lenticule diameter difference on the visual outcome and higher-order aberrations in SMILE: $0.4 \mathrm{~mm}$ versus $1.0 \mathrm{~mm}$. J Ophthalmol. 2017;2017:8259546.

31. Moshirfar M, McCaughey MV, Reinstein DZ, Shah R, Santiago-Caban L, Fenzl CR. Small-incision lenticule extraction. J Cataract Refract Surg. 2015 Mar;41(3):652-65. 
32. Miruna N, Andrei F, Vasile FM, Rotaru E. Smile--the next generation of laser vision correction. Rom J Ophthalmol. 2016;60(1):6-8

33. Moshirfar M, Albarracin JC, Desautels JD, Birdsong OC, Linn SH, Hoopes PC. Ectasia following small-incision lenticule extraction (SMILE): A review of the literature. Clin Ophthalmol. 2017;11:1683-1688.
34. Moshirfar M, Bruner CD, Skanchy DF, Shah T. Hyperopic small-incision lenticule extraction. Curr Opin Ophthalmol. 2019 Jul;30(4):229-235.

35. Siedlecki J, Luft N, Keidel L, et al. Variation of lenticule thickness for SMILE in low myopia. J Refract Surg. 2018;34(7):453-459.

This work is licensed under a Creative Commons Attribution-NonCommercial 3.0 Unported License which allows users to read, copy, distribute and make derivative works for non-commercial purposes from the material, as long as the author of the original work is cited properly. 